\title{
Persistent Structural Modifications in Nucleus Accumbens and Prefrontal Cortex Neurons Produced by Previous Experience with Amphetamine
}

\author{
Terry E. Robinson ${ }^{1}$ and Bryan Kolb² \\ ${ }^{1}$ Department of Psychology and Neuroscience Program, The University of Michigan, Ann Arbor, Michigan 48109, and \\ 2Department of Psychology, University of Lethbridge, Lethbridge, Alberta T1K 3M4, Canada
}

Experience-dependent changes in behavior are thought to involve structural modifications in the nervous system, especially alterations in patterns of synaptic connectivity. Repeated experience with drugs of abuse can result in very long-lasting changes in behavior, including a persistent hypersensitivity (sensitization) to their psychomotor activating and rewarding effects. It was hypothesized, therefore, that repeated treatment with the psychomotor stimulant drug amphetamine, which produces robust sensitization, would produce structural adaptations in brain regions that mediate its psychomotor activating and rewarding effects. Consistent with this hypothesis, it was found that amphetamine treatment altered the morphology of neurons in the nucleus accumbens and prefrontal cortex. Exposure to amphetamine produced a long-lasting ( $>1$ month) increase in the length of dendrites, in the density of dendritic spines, and in the number of branched spines on the major output cells of the nucleus accumbens, the medium spiny neurons, as indicated by analysis of Golgi-stained material. Amphetamine treatment produced similar effects on the apical (but not basilar) dendrites of layer III pyramidal neurons in the prefrontal cortex. The ability of amphetamine to alter patterns of synaptic connectivity in these structures may contribute to some of the long-term behavioral consequences of repeated amphetamine use, including amphetamine psychosis and addiction.

Key words: amphetamine; sensitization; Golgi staining; nucleus accumbens; prefrontal cortex; plasticity; psychostimulant drugs
The repeated intermittent administration of many drugs of abuse results in a progressive increase in their psychomotor activating and rewarding effects, a phenomenon known as behavioral sensitization (Segal and Schuckit, 1983; Robinson and Becker, 1986; Robinson and Berridge, 1993). Behavioral sensitization is interesting for at least two reasons. First, it is a compelling example of experience-dependent plasticity. Sensitized animals remain hypersensitive to the psychomotor activating and rewarding effects of drugs for months to years (Paulson et al., 1991; Valadez and Schenk, 1994). Second, the neuroadaptations that underlie behavioral sensitization may contribute to drug-induced psychopathology in humans (Segal and Schuckit, 1983; Robinson and Becker, 1986; Robinson and Berridge, 1993; Berridge and Robinson, 1995).

The psychomotor activating and rewarding effects of psychostimulant drugs are mediated by their actions on the nucleus accumbens or related circuitry, especially afferents arising from dopamine-containing cells in the ventral tegmental area and glutamate-containing cells in the prefrontal cortex (Wise and Bozarth, 1987; Koob and Bloom, 1988; Carlezon and Wise, 1996). For this reason, studies on the neurobiological basis of behavioral sensitization have focused on the accumbens system.

\footnotetext{
Received June 23, 1997; revised Aug. 5, 1997; accepted Aug. 13, 1997.

This work was supported by grants from the National Institute on Drug Abuse and from the National Science and Engineering Research Council of Canada. We thank G. Gorney and R. Gibb for excellent technical assistance and K. C. Berridge, K. Browman, and K. M. Harris for helpful comments on an earlier draft of this manuscript.

Correspondence should be addressed to Dr. Terry E. Robinson, Biopsychology Program, Department of Psychology, The University of Michigan, 525 East University (East Hall), Ann Arbor, MI 48109.

Copyright (C) 1997 Society for Neuroscience $\quad 0270-6474 / 97 / 178491-07 \$ 05.00 / 0$
}

A number of neurochemical and neurophysiological correlates have been found, including an increase in the ability of amphetamine, cocaine, or morphine to enhance the overflow of dopamine in the nucleus accumbens (Robinson and Becker, 1986; Kalivas and Stewart, 1991) and dopamine $\mathrm{D}_{1}$ receptor hypersensitivity (Henry and White, 1991; White and Wolf, 1991). However, experience-dependent changes in behavior that can persist for months, years, or a lifetime may not be mediated simply by changes in neurotransmitter dynamics or receptor characteristics, because of the rapid turnover of proteins involved in these processes (Greenough, 1984). Researchers who study other forms of experience-dependent plasticity, such as that involved in learning, environmental enrichment, or recovery of function, have emphasized that truly persistent alterations in behavior are probably mediated by structural modifications in neural circuitry, especially alterations in patterns of synaptic connectivity (Greenough, 1984; Greenough and Bailey, 1988; Kolb et al., 1997b).

Thus, the purpose of the experiment reported here was to determine whether an amphetamine treatment regimen known to produce robust and persistent behavioral sensitization would produce structural modifications in the nervous system similar to those seen in association with other forms of experiencedependent plasticity. Because the behaviors that are sensitized by amphetamine are mediated in part by drug actions in the nucleus accumbens and prefrontal cortex, we hypothesized that neurons in these brain regions would be affected. A common approach to examine the impact of experience on synaptic organization is to use Golgi-stained material to quantify the structure of dendrites (Greenough, 1984; Greenough et al., 1990; Kolb et al., 1997b). In adult animals there is a good relationship between the dendritic surface available for synapses and the number of synapses on a 


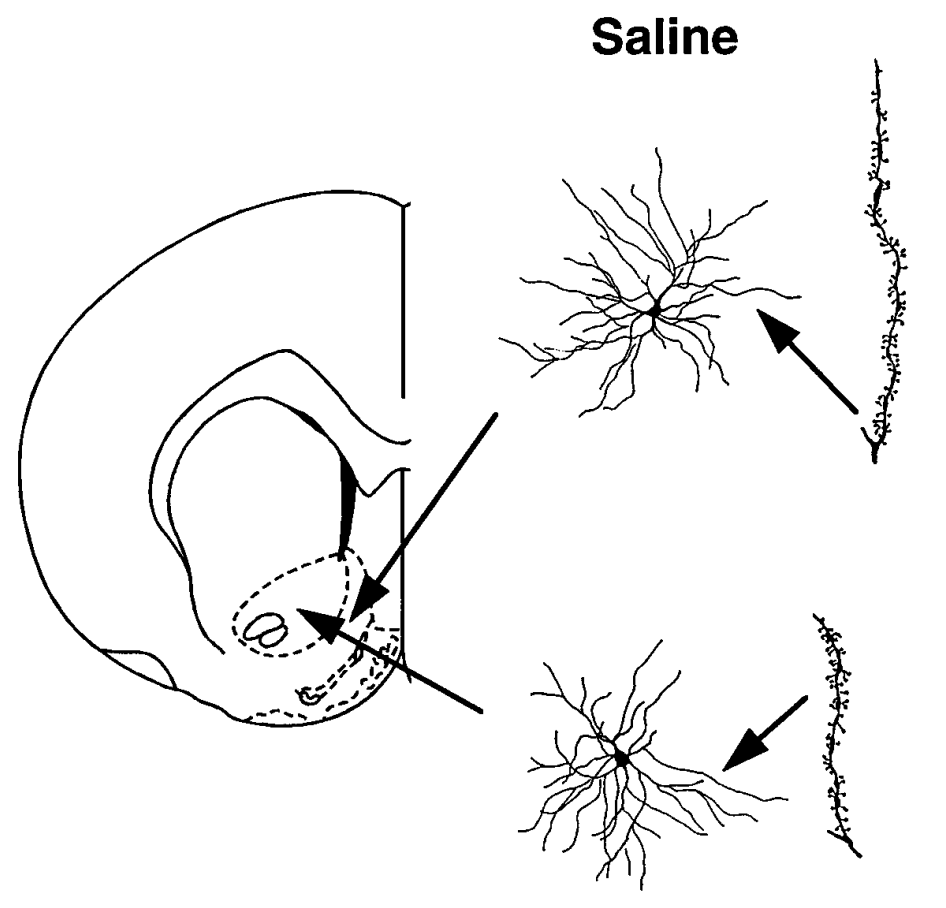

Amphetamine
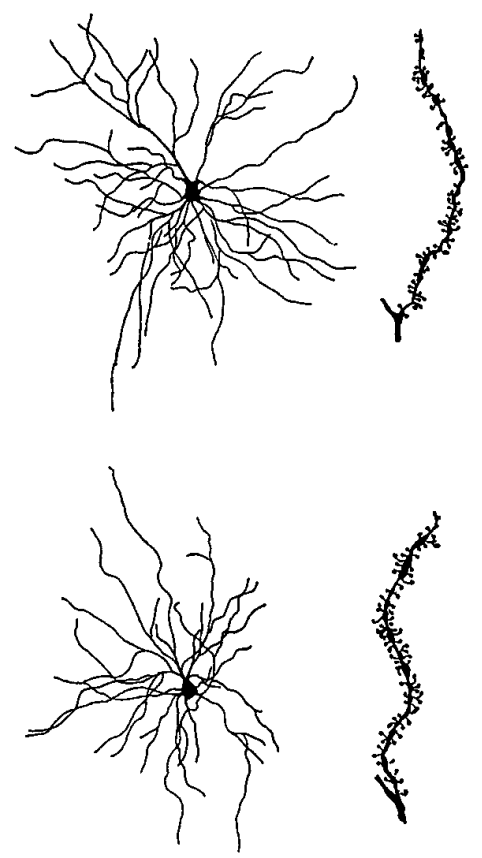

Figure 1. Camera lucida drawings of medium spiny neurons in the shell (top) and core (bottom) regions of the nucleus accumbens of salineand amphetamine-pretreated rats. These cells were selected for illustration because their values were closest to the group average of any cells studied. The drawing to the right of each cell represents a dendritic segment used to calculate spine density. Coronal drawing is adapted from Paxinos and Watson (1997).

neuron (Greenough and Chang, 1988; Harris and Kater, 1994; Purves, 1994). Therefore, we used this method to quantify the effect of repeated amphetamine treatment on the dendritic structure of medium spiny neurons in the nucleus accumbens and layer III pyramidal neurons in the prefrontal cortex, the major output neurons in these brain regions.

\section{MATERIALS AND METHODS}

Male Sprague Dawley rats weighing 255-355 gm were housed individually with food and water available ad libitum. Half of the animals $(n=5)$ were pretreated with D-amphetamine sulfate using an escalating-dose regimen similar to that described previously (Paulson et al., 1991). Each rat received two intraperitoneal injections per day, $\sim 8 \mathrm{hr}$ apart, for 5 consecutive days, followed by 2 drug-free days for a total of 5 weeks, beginning with $1 \mathrm{mg} / \mathrm{kg}$ (weight of the salt) and escalating to $8 \mathrm{mg} / \mathrm{kg}$ for the last $4 \mathrm{~d}$ of treatment. Control animals $(n=5)$ received $0.9 \%$ saline. All injections were given in the home cage. The animals were then left undisturbed for $38 \mathrm{~d}$.

This treatment regimen was used because we have characterized its behavioral effects and some of its neurochemical effects in a series of previous studies (Robinson and Camp, 1987; Paulson et al., 1991; Paulson and Robinson, 1995, 1996). This treatment regimen produces very robust behavioral sensitization that persists for up to 1 year after the discontinuation of drug treatment. It is also important to note that it is not neurotoxic to dopamine neurons, as is the case with continuous amphetamine administration or with much higher dose regimens. As might be expected, amphetamine neurotoxicity is associated with signs of cellular degeneration (Ricaurte et al., 1982; Ryan et al., 1990).

Thirty-eight days after the last treatment with amphetamine or saline, the rats were deeply anesthetized with sodium pentobarbital and then were perfused intracardially with $0.9 \%$ saline. The brains were removed and prepared for Golgi-Cox staining. Traditional Golgi-Cox methods provide capricious staining of spines, but the modified method used here allows consistent visualization of spines (Kolb and McClimans, 1986; Kolb et al., 1997a). Briefly, the brains were first placed in Golgi-Cox solution for $14 \mathrm{~d}$ followed by $3 \mathrm{~d}$ in $30 \%$ sucrose. They then were cut into $200 \mu \mathrm{m}$ coronal sections using a vibratome and stained (Kolb and
McClimans, 1986). To be included in the analysis, the dendritic tree of a cell had to be well impregnated and not obscured with stain precipitations, blood vessels, or astrocytes, and the dendritic fields had to appear primarily intact and visible in the plane of section. The relevant brain regions were identified at low power $(100 \times)$, and five layer III pyramidal cells from each hemisphere were drawn using a camera lucida (at $250 \times$ ) in cortical areas $\mathrm{Cg} 3$ (prefrontal), Par 1 (parietal), and Oc 1 (occipital), as defined by Zilles (1985). Medium spiny neurons in the core and shell of the nucleus accumbens were identified and drawn in the same manner.

A Sholl analysis (Sholl, 1981) of ring intersections was used to estimate dendritic length, and for cortical cells, dendritic length was also estimated using the branch order method (Greenough and Chang, 1985). Spine density was quantified by counting spines on one third-order terminal tip from the basilar and apical dendrites of each pyramidal cell and on one terminal tip of each accumbens cell. A length of dendrite $(>10 \mu \mathrm{m})$ was traced $(1000 \times)$, and the exact length of the dendritic segment was calculated. In a second analysis to examine the frequency of branched spines on nucleus accumbens cells, this procedure was repeated, but dendritic segments were drawn at $2000 \times$, and both total spines and spines with more than one head were counted. Branched spines were defined as described by Comery et al. (1996), that is, spines with more than one head clearly connected to a common shaft. Thus, this analysis represents both a replication of the initial analysis, because different dendritic segments were drawn, and an extension to determine whether amphetamine had a disproportionate effect on the number of branched spines (i.e., those with multiple heads), as reported recently for rats raised in a complex environment (Comery et al., 1996).

Cell selection and drawing were done by a person blind to treatment conditions. Statistical analyses were performed by averaging across cells per hemisphere, and group differences were assessed using Student's $t$ tests.

\section{RESULTS}

Figure 1 shows camera lucida drawings of representative medium spiny neurons in the core and shell regions of the nucleus accumbens from saline- and amphetamine-pretreated rats. It is evident from inspection of Figure 1 that there was an increase in dendritic 
length and spine density in amphetamine-pretreated rats, an impression that was verified by quantitative analyses (Fig. $2 A$ ). Dendritic length was increased by $16.5 \%$ in the core of the accumbens $(t=8.77 ; p=0.0083)$ and by $8.2 \%$ in the shell of the accumbens $(t=4.37 ; p=0.051)$. Spine density was increased by $19.6 \%$ in the core $(t=108.8 ; p<0.0001)$ and by $25.9 \%$ in the shell $(t=179.3 ; p<0.0001)$. Figure 3 shows camera lucida drawings of representative dendritic segments from shell and core cells magnified $2000 \times$. These were used to examine the frequency of branched spines. This analysis replicated the initial one, because, as shown in Figure 3, total spine density was increased in amphetamine-pretreated rats by $32.1 \%$ in the shell of the accumbens $(t=241 ; p<0.0001)$ and by $27.8 \%$ in the core $(t=287 ; p<$ $0.0001)$. More interestingly, there was a disproportionate increase in the number of branched spines (Fig. 3). In amphetaminepretreated rats, the number of branched spines was increased by $203.6 \%$ in the shell of the accumbens $(t=157 ; p<0.0001)$ and by $117.6 \%$ in the core $(t=124 ; p<0.0001)$. Thus, in salinepretreated rats, branched spines comprised 6.9 and $8.9 \%$ of total spines in the shell and core regions, respectively, whereas in amphetamine-pretreated rats, branched spines comprised 16 and $15.2 \%$ of total spines in these regions.

Another effect that is obvious from Figure 3 is that the dendritic segments from amphetamine-pretreated rats were thicker than those from saline-pretreated rats. This effect was not quantified, but it was clearly evident in all of the accumbens cells examined.

The only other region examined in which amphetamine pretreatment altered dendritic length was the prefrontal cortex (Figs. $2 B, 4)$, and only apical dendrites were altered. Amphetamine pretreatment increased apical dendritic length of layer III prefrontal cortical neurons by $12.4 \%(t=4.57 ; p=0.048)$, whereas basilar dendritic length was unchanged (Fig. $2 B ; t=0.15$ ). Similarly, spine density was increased on apical dendrites $(t=80.8$; $p<0.0001)$ but not on basilar dendrites $(t=1.41)$ of prefrontal cortex cells (Fig. 2B).

In the two other cortical regions examined, there was no effect of amphetamine pretreatment on dendritic length for either apical or basilar dendrites (Fig. 2C,D; for the parietal cortex, $t$ values $<0.3$; for the occipital cortex, $t$ values $<0.5$ ). There was, however, a significant decrease in spine density in both the parietal and occipital cortices (Fig. 2C,D; for parietal apical, $t=197.0$ and $p<0.0001$; for parietal basilar, $t=73.25$ and $p<0.0001$; for occipital apical, $t=140.7$ and $p<0.0001$; for occipital basilar, $t=$ 41.14 and $p<0.0001$ ).

A branch order analysis on cortical cells in all three regions yielded the same pattern of results as seen with the Sholl analysis reported above (data not shown).

\section{DISCUSSION}

Repeated exposure to psychostimulant drugs results in many neurobehavioral adaptations that outlast the acute effects of the drug. Some of these are relatively transient, such as those associated with withdrawal syndromes, and others are remarkably persistent, such as those associated with behavioral sensitization. The amphetamine treatment regimen used here has been shown many times both to produce a withdrawal syndrome (nocturnal hypoactivity) and to produce behavioral sensitization (Robinson and Camp, 1987; Paulson et al., 1991; Paulson and Robinson, 1995, 1996). Withdrawal symptoms subside $\sim 1$ week after the discontinuation of drug treatment, at which time animals begin to express hypersensitivity to a drug challenge (Paulson et al.,
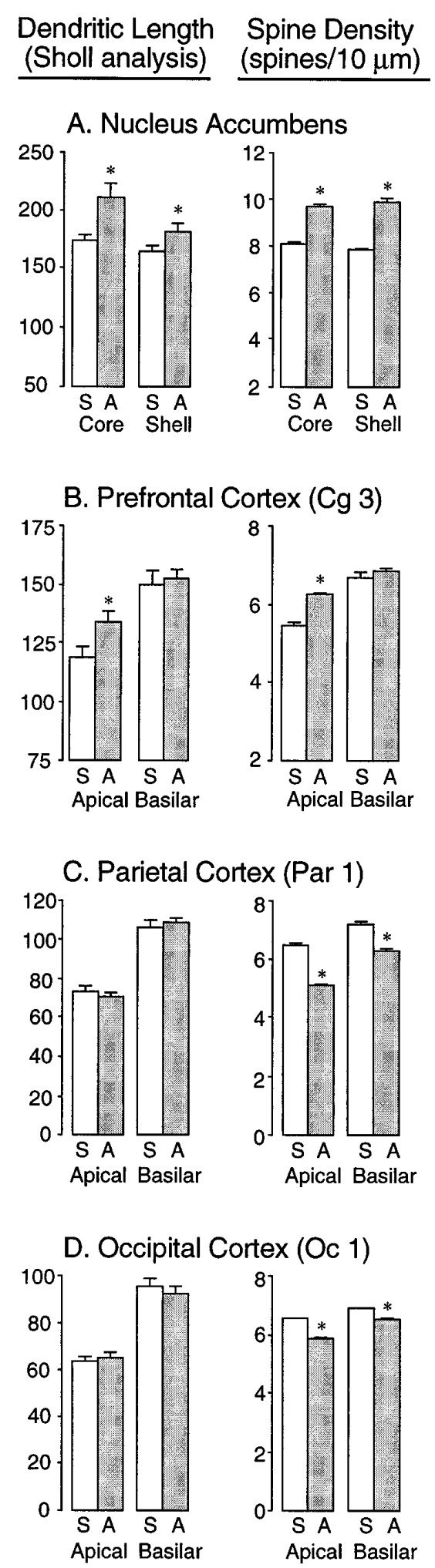

Figure 2. Quantitative analysis of dendritic length (left) and spine density (right) in the nucleus accumbens shell and core subregions $(A)$, the prefrontal cortex $(B)$, the parietal cortex $(C)$, and the occipital cortex $(D)$ of rats treated with saline (indicated by $S$ ) or amphetamine (indicated by $A)$. The bars represent the values of mean plus SEM. Dendritic length was estimated using a Sholl analysis, and therefore the numbers indicate ring intersections. Spine density refers to the mean total number of spines per $10 \mu \mathrm{m}$ of dendrite. Asterisks indicate a significant difference between saline- and amphetamine-pretreated groups (see Results). 

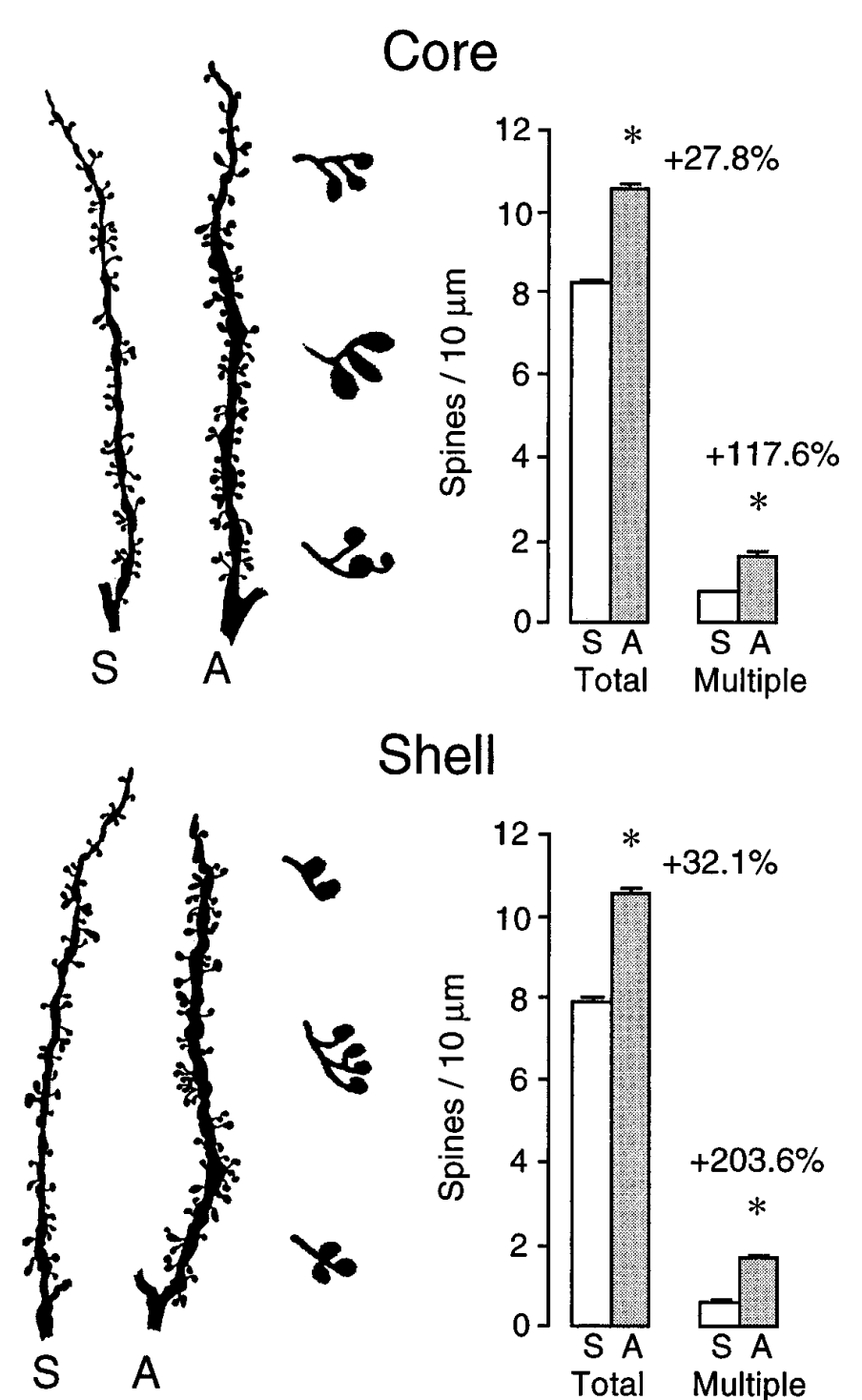

Shell

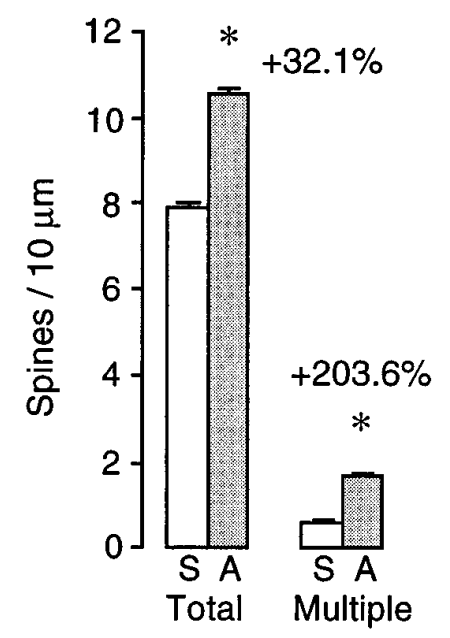

Figure 3. Left, Camera lucida drawings of representative dendritic segments from medium spiny neurons in the core (top) and shell (bottom) subregions of the nucleus accumbens of rats pretreated with saline $(S)$ and amphetamine $(A)$. These drawings were magnified $2000 \times$ and were used to count branched spines. Enlarged examples of different forms of branched spines that were observed in amphetamine-pretreated animals are shown in the third column from the left. Right, Bar graphs showing the results of the quantitative analyses. Asterisks indicate that in amphetamine-pretreated rats there was a significant increase in total spines per $10 \mu \mathrm{m}$ of dendrite and in the number of spines with multiple heads (branched spines).

1991). Sensitization is fully evident 2 weeks to 1 month after the last drug treatment and can persist at least 1 year (Paulson et al., 1991).

Relatively transient behavioral sequelae associated with withdrawal may be caused by transient adaptations in those neurochemical processes involved in mediating acute drug effects. However, very persistent experience-dependent changes in behavior are thought to involve a different class of adaptations, changes in synaptic connectivity (Greenough, 1984). Consistent with this hypothesis, we found that 1 month after the last treatment with amphetamine there was an increase in dendritic surface and in the number of dendritic spines on medium spiny neurons located in the shell and core subregions of the nucleus accumbens and on the apical (but not basilar) dendrites of layer III pyramidal cells in the prefrontal cortex.

Changes in dendritic structure reflected in Golgi material are considered strong evidence of changes in synaptic connectivity. It has been estimated that $>90 \%$ of all excitatory synapses are on dendritic spines, and in adult animals, as the amount of dendritic surface increases so does the number of synaptic contacts (Harris and Kater, 1994). A strong relationship between measures of dendritic structure and synaptic input has been confirmed in studies using electron microscopy (EM) to quantify directly synaptic density. For example, Greenough and colleagues have shown that in rats raised in complex environments, or subjected to various learning experiences, increases in the dendritic surface of cortical neurons assessed using Golgi-stained material are accompanied by increases in the number of synapses per neuron assessed with EM (Greenough and Bailey, 1988; Greenough et al., 1990). More recently, Purves (1994) and colleagues have shown a direct relationship between the number of dendritic branches and the number of synapses in sympathetic and parasympathetic ganglia. Of course, without ultrastructural studies we cannot be certain that the increase in dendritic surface and spines found here is accompanied by an increase in synaptic contacts, but normally in adult rats nearly all spines in the cortex and striatum have a synaptic contact (Gray, 1959; Peters and Feldman, 1976; Wilson et al., 1983). If this is also true for amphetamine-sensitized rats, it would seem that repeated exposure to amphetamine produces fundamental alterations in the pattern and number of synaptic connections on the major output neurons of the nucleus accumbens and prefrontal cortex.

The increase in the number of branched spines is especially intriguing, because this may provide a very effective mechanism to enhance synaptic efficacy (Trommald et al., 1990). Very little is known about branched spines (Harris and Stevens, 1988; Chicurel and Harris, 1992), but an increase in branched spines has been reported in dentate granule cells after long-term potentiation (Trommald et al., 1990) or kindling produced by medial perforant path stimulation (Geinisman et al., 1989) and in the dorsolateral striatum after rearing in a complex environment (Comery et al., 1996). Trommald et al. (1990) reported that after long-term potentiation both branches of a branched spine were always associated with normal-appearing presynaptic active zones, and in all cases each branch was innervated by a different axon. Whether this will be the case here remains to be tested, but as pointed out by Trommald et al. (1990), the addition of a second spine head to an existing spine may provide a very efficient way to double the input effect at a specific location.

The increase in spine density and the number of branched spines produced by past exposure to amphetamine was found on the terminal dendrites of medium spiny neurons and pyramidal cells. We need to consider, therefore, the nature of the synaptic inputs onto this portion of the dendritic tree. For medium spiny neurons, the most complete information is for cells in the dorsal striatum. The distal dendrites of medium spiny neurons receive inputs primarily from two structures extrinsic to the striatum (Smith and Bolam, 1990). The heads of spines are innervated by boutons that form an asymmetric contact; these boutons are thought to arise in the neocortex and to use an excitatory amino acid (EAA), probably glutamate, as their transmitter. Approximately $50 \%$ of these spines also receive a dopaminergic input that forms a symmetric contact with either the neck of the spine or an area nearby on the shaft of the dendrite (Smith and Bolam, 1990; 


\section{Saline Amphetamine}

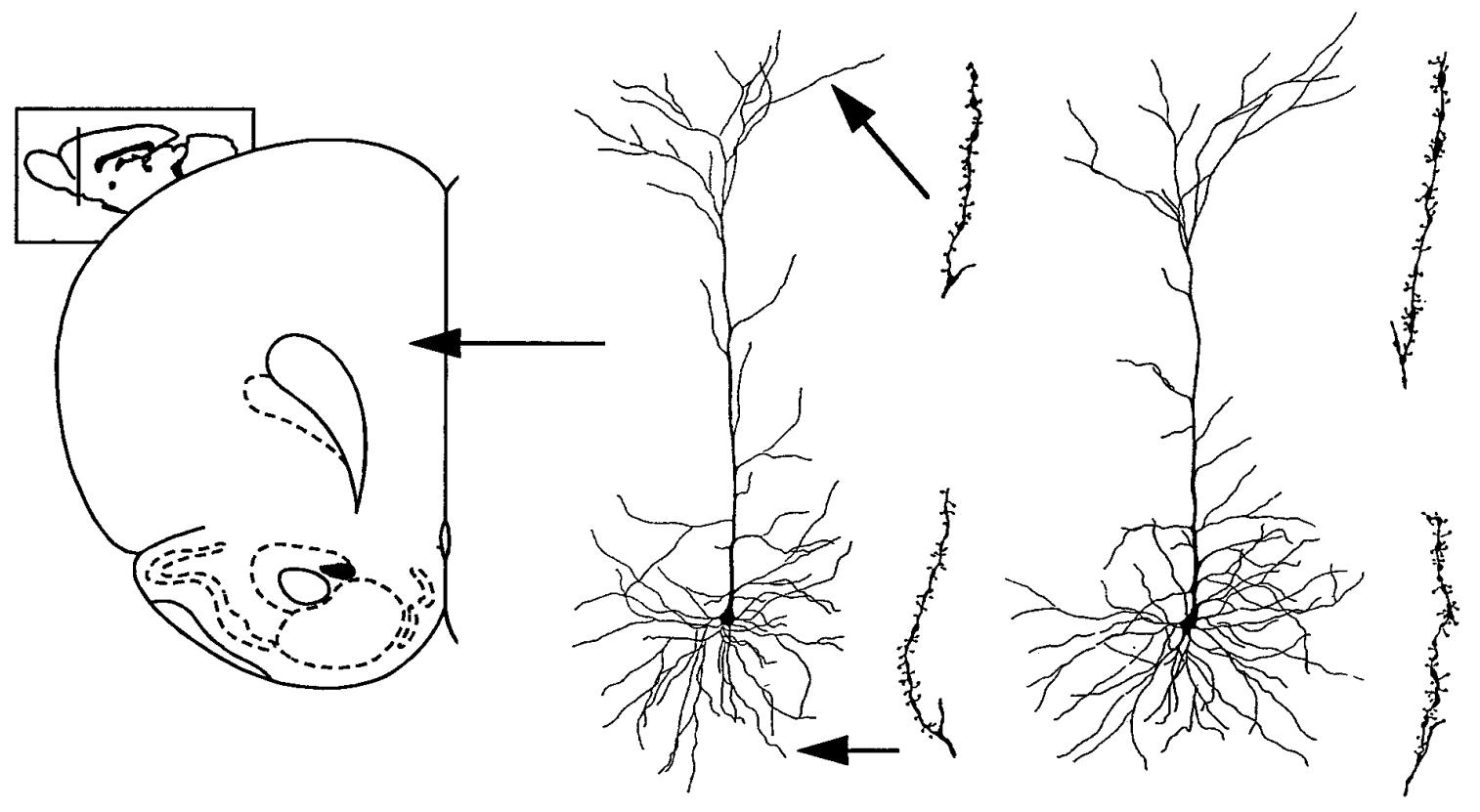

Figure 4. Camera lucida drawings of representative layer III pyramidal cells in the prefrontal cortex ( $\operatorname{area} \mathrm{Cg} 3$ ) of saline- and amphetamine-pretreated rats. The drawing to the right of each cell represents an apical or basilar dendritic segment used to calculate spine density. Coronal drawing is adapted from Paxinos and Watson (1997).

Groenewegen et al., 1991). Thus, a fundamental unit on the distal portion of medium spiny neuron dendrites is a "triad" consisting of a spine that receives both an excitatory (asymmetric) input from the cortex and an inhibitory (symmetric) input from dopamine-containing cells in the tegmentum (Smith and Bolam, 1990; Groenewegen et al., 1991). More proximal portions of the dendrites and the cell body receive inputs that arise primarily intrinsic to the striatum.

This triad structure is also found in the nucleus accumbens and the prefrontal cortex (Goldman-Rakic et al., 1989, 1992; Smith and Bolam, 1990; Berger et al., 1991; Groenewegen et al., 1991). In the nucleus accumbens, asymmetric (presumed EAA) inputs arise from structures such as the prefrontal cortex and hippocampus (Totterdell and Smith, 1989; Sesack and Pickel, 1990, 1992; Smith and Bolam, 1990; Groenewegen et al., 1991). Similarly, in the prefrontal cortex, spines on pyramidal cells that have presumed dopaminergic synapses also have asymmetric (presumed EAA) synapses (Berger et al., 1991; Goldman-Rakic et al., 1992). It has been suggested, therefore, that in both the striatum and neocortex, triads provide the structural means by which dopamine modulates or gates the major excitatory drive produced by EAA inputs onto output neurons (Smith and Bolam, 1990; Berger et al., 1991; Groenewegen et al., 1991; Goldman-Rakic et al., 1992). This convergence of dopaminergic and presumed glutaminergic inputs at the site of morphological adaptation reported here is especially intriguing, because both of these neurotransmitter systems have been prominently implicated in amphetamine sensitization. Psychomotor sensitization to amphetamine is prevented by cotreatment with either dopamine or glutamate antagonists (Kalivas and Stewart, 1991; White and Wolf, 1991). Furthermore, psychostimulant sensitization is associated with alterations in the responsiveness of nucleus accumbens neurons to both dopamine and glutamate agonists (Henry and White, 1991; White et al.,
1995). Thus, changes in triads, the sites of convergence of dopamine and glutamate inputs onto medium spiny neurons, may provide the structural basis for sensitization-related alterations in the responsiveness of nucleus accumbens neurons to both dopamine and glutamate inputs. The structural adaptations reported here would require new protein synthesis, so it is also important to note that sensitization is prevented by cotreatment with protein synthesis inhibitors (Robinson, 1991; Karler et al., 1993).

Figure 5 summarizes one proposed site of morphological plasticity associated with repeated amphetamine treatment, the triad found on the distal dendrites of medium spiny neurons and prefrontal pyramidal cells. Amphetamine pretreatment increased dendritic length, increased spine density, and increased the number of branched spines in those regions in which triads are prevalent. These latter two modifications are represented diagrammatically in Figure $5 B$. Figure 5 also indicates that the relationship between these postsynaptic modifications and presumed new patterns of synaptic connectivity is not known, and there are a number of ways the triad arrangement could be altered (for discussion, see Fig. 5). Of course, there could also be structural changes in the presynaptic boutons (e.g., Uranova et al., 1989) or other changes in dendritic structure (Harris and Kater, 1994). Whatever the case, the present results suggest that repeated amphetamine treatment can produce persistent morphological adaptations in the basic "processing unit" associated with distal dendritic branches of output neurons in the nucleus accumbens and prefrontal cortex, the synaptic triad.

In closing, it is interesting to speculate about what role these structural adaptations might play in some of the long-term behavioral consequences of repeated exposure to psychomotor stimulant drugs in humans. One consequence of repeated amphetamine use is amphetamine psychosis, a syndrome characterized by paranoid schizophrenic-like symptoms (Segal and Schuckit, 


\section{$\begin{array}{lll}\text { A. Control } & \text { B. Amphetamine }\end{array}$}

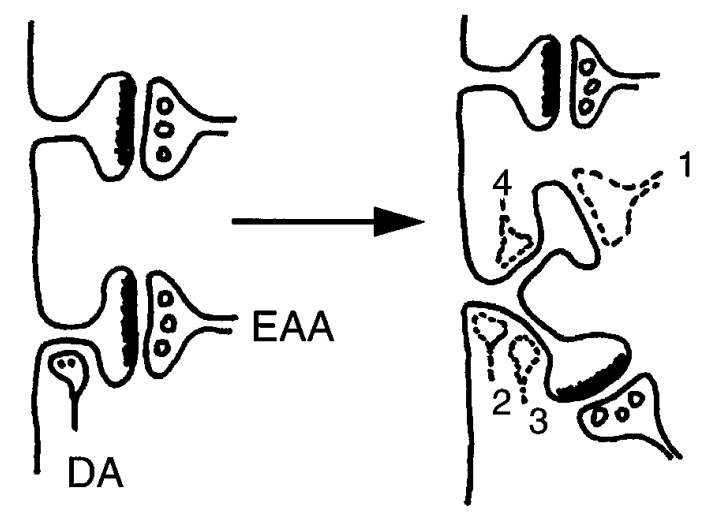

Figure 5. A schematic illustration of the effect of amphetamine pretreatment on spine density in the nucleus accumbens. $A$, Two spines on a distal dendrite of a medium spiny neuron are illustrated. In the striatum, spine heads on distal dendrites are innervated by presumed EAA inputs from the cortex (e.g., prefrontal cortex or hippocampus in the case of the accumbens) that form asymmetric contacts. Approximately half of these spines also receive a dopaminergic $(D A)$ input onto the neck of the spine or nearby onto the dendritic shaft (Smith and Bolam, 1990; Groenewegen et al., 1991). The lower spine in $A$ illustrates one of these triads. After amphetamine treatment, there was an increase in dendritic surface and spine density in the regions occupied by triads. $B$, Amphetamine treatment produced an especially large increase in the number of branched spines. What is not known, of course, is the relationship between this altered postsynaptic surface and patterns of synaptic connectivity (indicated by the dashed terminals). There are a number of possibilities. It is possible, of course, that the additional spines are unoccupied. This seems unlikely because previous studies on experience-dependent plasticity suggest that in adult animals spines are not typically vacant. Alternatively, the heads of the additional spines could receive new asymmetric contacts (dashed terminal 1 in $B$ ). If normal proportions were maintained, it would be expected that approximately half of these new spines would receive both a symmetric dopaminergic input onto their neck and an asymmetric EAA input onto the spine head. The other half of the new spines would receive only an asymmetric contact. But there are a number of ways the DA inputs could be arranged. One very interesting possibility is that the original DA input would remain near the base of the common spine neck, near or on the dendritic shaft (dashed terminal 2), where it could modulate both EAA inputs onto the spine heads. This might provide a mechanism by which DA could exert greater modulatory control over the excitatory drive onto medium spiny neurons without any "sprouting" of additional DA terminals. This idea is consistent with observations that nucleus accumbens cells in sensitized rats are more sensitive to DA agonists (Henry and White, 1991) but less sensitive to glutamate (White et al., 1995). Alternatively, the DA input on the original spine could move out to the neck of the original branch (dashed terminal 3). If there was no additional DA terminal added to the neck of the new branched spine (dashed terminal 4 ), this could result in a decrease in dopaminergic control over EAA inputs at this location because the asymmetric input onto the new spine head would be "unopposed" by a symmetric input onto the same spine. An unlikely possibility is that there is an increase in the number of dopaminergic synapses onto spines that have no EAA input. This would be unusual, because in normal animals symmetric contacts are almost always accompanied by an asymmetric contact (Smith and Bolam, 1990; Groenewegen et al., 1991). Finally, some spines on the distal dendrites could become occupied by inputs that normally are not found there, for example, from sources intrinsic to the nucleus accumbens. To determine which of these, or other, scenarios is correct will require detailed ultrastructural studies. Whatever the new pattern of synaptic connectivity, it is obvious that there are many ways by which the structural modifications reported here could produce enduring changes in the effects of DA and glutamate neurotransmitters on the excitability of nucleus accumbens medium spiny neurons (and prefrontal pyramidal neurons).
1983; Robinson and Becker, 1986). It has been suggested that aberrant dopamine-glutamate interactions in corticostriatal thalamocortical loops play a role in the pathophysiology of schizophrenia (Carlsson and Carlsson, 1990); therefore, amphetamine-induced changes in dopamine-glutamate triads in the prefrontal cortex and striatum could contribute to the development of amphetamine psychosis. Another consequence of repeated exposure to psychostimulant drugs is addiction. Sensitization-related neuroadaptations in brain reward systems may be important in the development of addiction (Robinson and Berridge, 1993; Berridge and Robinson, 1995), and the medium spiny neurons have been proposed to "serve as the final common path of opiate and psychostimulant reward" (Carlezon and Wise, 1996, p 3120). It is possible, therefore, that the compulsive pattern of drug-taking behavior that characterizes addiction may develop in part because of the ability of some drugs to alter patterns of synaptic connectivity in the very brain regions that mediate drug reward. Of course, how they do so, what role structural modifications actually play in mediating behavioral change, including sensitization, and whether other drugs of abuse produce similar adaptations will require further study. Although we are left with many unanswered questions, the results reported here suggest a promising new avenue for exploring drug-dependent plasticity in the nervous system.

\section{REFERENCES}

Berger B, Gaspar P, Verney C (1991) Dopaminergic innervation of the cerebral cortex: unexpected differences between rodents and primates. Trends Neurosci 14:21-27.

Berridge KC, Robinson TE (1995) The mind of an addicted brain: neural sensitization of wanting versus liking. Curr Direct Psychol Sci 4:71-76.

Carlezon WA, Wise RA (1996) Rewarding actions of phencyclidine and related drugs in nucleus accumbens shell and frontal cortex. J Neurosci 16:3112-3122.

Carlsson M, Carlsson A (1990) Interactions between glutamatergic and monoaminergic systems within the basal ganglia-implications for schizophrenia and Parkinson's disease. Trends Neurosci 13:272-276.

Chicurel ME, Harris KM (1992) Three-dimensional analysis of the structure and composition of CA3 branched dendritic spines and their synaptic relationships with mossy fiber boutons in the rat hippocampus. J Comp Neurol 325:169-182.

Comery TA, Stamoudis CX, Irwin SA, Greenough WT (1996) Increased density of multiple-head dendritic spines on medium-sized spiny neurons of the striatum in rats reared in a complex environment. Neurobiol Learn Mem 66:93-96.

Geinisman Y, Morell F, de Toledo-Morell L (1989) Perforated synapses on double-headed dendritic spines: a possible substrate of synaptic plasticity. Brain Res 480:326-329.

Goldman-Rakic PS, Leranth C, Williams MS, Mons N, Geffard M (1989) Dopamine synaptic complex with pyramidal neurons in primate cerebral cortex. Proc Natl Acad Sci USA 86:9015-9019.

Goldman-Rakic PS, Lidow MS, Smiley JF, Williams MS (1992) The anatomy of dopamine in monkey and human prefrontal cortex. J Neural Transm 36:163-177.

Gray EG (1959) Axo-somatic and axo-dendritic synapses of the cerebral cortex: an electron microscope study. J Anat 93:420-433.

Greenough WT (1984) Structural correlates of information storage in the mammalian brain: a review and hypothesis. Trends Neurosci 7:229-233.

Greenough WT, Bailey CH (1988) The anatomy of a memory: convergence of results across a diversity of tests. Trends Neurosci 11:142-147.

Greenough WT, Chang F-LF (1985) Synaptic structural correlates of information storage in mammalian nervous systems. In: Synaptic plasticity (Cotman CW, ed), pp 335-372. New York: Guilford.

Greenough WT, Chang F-LF (1988) Plasticity of synapse structure and pattern in the cerebral cortex. In: Cerebral cortex, (Peters A, Jones EG, eds), pp 391-440. New York: Plenum.

Greenough WT, Withers GS, Wallace CS (1990) Morphological changes in the nervous system arising from behavioral experience: what is the 
evidence that they are involved in learning and memory? In: The biology of memory, Symposia Medica Hoechst (Squire LR, Lindenlaub E, eds), pp 159-185. New York: Schattauder.

Groenewegen HJ, Berendse HW, Meredith GE, Haber SN, Voorn P, Wolters JG, Lohman AHM (1991) Functional anatomy of the ventral, limbic system-innervated striatum. In: The mesolimbic dopamine system: from motivation to action (Willner P, Scheel-Krüger J, eds), pp 19-59. New York: Wiley.

Harris KM, Kater SB (1994) Dendritic spines: cellular specializations imparting both stability and flexibility to synaptic function. Annu Rev Neurosci 17:341-371.

Harris KM, Stevens JK (1988) Dendritic spines of rat cerebellar Purkinje cells: serial electron microscopy with reference to their biophysical characteristics. J Neurosci 8:4455-4469.

Henry DJ, White FJ (1991) Repeated cocaine administration causes persistent enhancement of D1 dopamine receptor sensitivity within the rat nucleus accumbens. J Pharmacol Exp Ther 258:882-890.

Kalivas PW, Stewart J (1991) Dopamine transmission in the initiation and expression of drug- and stress-induced sensitization of motor activity. Brain Res Rev 16:223-244.

Karler R, Finnegan KT, Calder LD (1993) Blockade of behavioral sensitization to cocaine and amphetamine by inhibitors of protein synthesis. Brain Res 603:19-24.

Kolb B, McClimans J (1986) Cryostat sectioning of Golgi-Cox tissue. Stain Tech 61:379-380.

Kolb B, Gorny G, Cote S, Ribeiro-da-Silvan A, Cuello AC (1997a) Nerve growth factor stimulates growth of cortical pyramidal neurons in young adult rats. Brain Res 761:289-294.

Kolb B, Forgie M, Gibb R, Gorny G, Rowntree S (1997b) Age, experience and the changing brain. Neurosci Biobehav Rev, in press.

Koob GF, Bloom FE (1988) Cellular and molecular mechanisms of drug dependence. Science 242:715-723.

Paulson PE, Robinson TE (1995) Amphetamine-induced timedependent sensitization of dopamine neurotransmission in the dorsal and ventral striatum: a microdialysis study in behaving rats. Synapse 19:56-65.

Paulson PE, Robinson TE (1996) Regional differences in the effects of amphetamine withdrawal on dopamine dynamics in the striatum: analysis of circadian patterns using automated on-line microdialysis. Neuropsychopharmacology 14:325-337.

Paulson PE, Camp DM, Robinson TE (1991) The time course of transient behavioral depression and persistent behavioral sensitization in relation to regional brain monoamine concentrations during amphetamine withdrawal in rats. Psychopharmacology (Berl) 103:480-492.

Paxinos G, Watson C (1997) The rat brain in stereotaxic coordinates, compact third edition. New York: Academic.

Peters A, Feldman ML (1976) The projection of the lateral geniculate nucleus to area 17 of the rat cerebral cortex. I. General description. J Neurocytol 5:63-84.

Purves D (1994) Neural activity and the growth of the brain. Cambridge, U.K.: Cambridge UP.

Ricaurte GA, Guillery RW, Seiden LS, Schuster CR, Moore RY (1982) Dopamine nerve terminal degeneration produced by high doses of methylamphetamine in the rat brain. Brain Res 235:93-103.

Robinson TE (1991) The neurobiology of amphetamine psychosis: evidence from studies with an animal model. In: Taniguchi symposia on brain sciences, Vol 14, Biological basis of schizophrenia (Nakazawa T, ed), pp 185-201. Tokyo: Japan Scientific Societies.

Robinson TE, Becker JB (1986) Enduring changes in brain and behavior produced by chronic amphetamine administration: a review and evaluation of animal models of amphetamine psychosis. Brain Res Rev 11:157-198.

Robinson TE, Berridge KC (1993) The neural basis of drug craving: an incentive-sensitization theory of addiction. Brain Res Rev 18:247-291.

Robinson TE, Camp DM (1987) Long-lasting effects of escalating doses of D-amphetamine on brain monoamines, amphetamine-induced stereotyped behavior and spontaneous nocturnal locomotion. Pharmacol Biochem Behav 26:821-827.

Ryan LJ, Linder JC, Martone ME, Groves PM (1990) Histological and ultrastructural evidence that D-amphetamine causes degeneration in neostriatum and frontal cortex of rats. Brain Res 518:67-77.

Segal DS, Schuckit MA (1983) Animal models of stimulant-induced psychosis. In: Stimulants: neurochemical, behavioral and clinical perspectives (Creese I, ed), pp 131-167. New York: Raven.

Sesack SR, Pickel VM (1990) In the rat medial nucleus accumbens, hippocampal and catecholaminergic terminals converge on spiny neurons and are in apposition to each other. Brain Res 527:266-279.

Sesack SR, Pickel VM (1992) Prefrontal cortical efferents in the rat synapse on unlabeled neuronal targets of catecholamine terminals in the nucleus accumbens septi and on dopamine neurons in the ventral tegmental area. J Comp Neurol 320:145-160.

Sholl DA (1981) The organization of the cerebral cortex. London: Methuen.

Smith AD, Bolam JP (1990) The neural network of the basal ganglia as revealed by the study of synaptic connections of identified neurons. Trends Neurosci 13:259-265.

Totterdell S, Smith AD (1989) Convergence of hippocampal and dopaminergic input onto identified neurons in the nucleus accumbens of the rat. J Chem Neuroanat 2:285-298.

Trommald M, Vaaland JL, Blackstad TW, Andersen P (1990) Dendritic spine changes in rat dentate granule cells associated with long-term potentiation. In: Neurotoxicity of excitatory amino acids (Guidotti A, ed), pp 163-174. New York: Raven.

Uranova NA, Klintzova AJ, Istomin VV, Haselhorst U, Schenk H (1989) The effects of amphetamine on synaptic plasticity in rat's medial prefrontal cortex. J Hirnforsch 30:45-50.

Valadez A, Schenk S (1994) Persistence of the ability of amphetamine preexposure to facilitate acquisition of cocaine self-administration. Pharmacol Biochem Behav 47:203-205.

White FJ, Wolf ME (1991) Psychomotor stimulants. In: The biological bases of drug tolerance and dependence (Pratt J, ed), pp 153-197. New York: Academic.

White FJ, Hu XT, Zhang XF, Wolf ME (1995) Repeated administration of cocaine or amphetamine alters neuronal responses to glutamate in the mesoaccumbens dopamine system. J Pharmacol Exp Ther 273:445-454.

Wilson CJ, Groves PM, Kitai ST, Linder JC (1983) Three-dimensional structure of dendritic spines in the rat neostriatum. J Neurosci 3:383-398.

Wise RA, Bozarth MA (1987) A psychomotor stimulant theory of addiction. Psychol Rev 94:469-492.

Zilles K (1985) The cortex of the rat. Berlin: Springer. 